\title{
miRNA expression profiles in chronic lymphocytic and acute lymphocytic leukemia
}

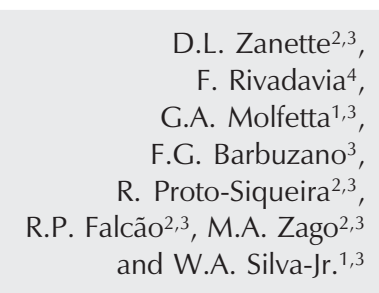

Correspondence

W.A. Silva-Jr.

Hemocentro de Ribeirão Preto

Rua Tenente Catão Roxo, 2501

14051-140 Ribeirão Preto, SP

Brasil

Fax: +55-16-2101-9309

E-mail: wilsonjr@usp.br

Research supported by FAPESP,

CNPq, CAPES, FINEP, and Applied

Biosystems, Brazil.

Received April 16, 2007

Accepted August 16, 2007

\author{
${ }^{1}$ Departamento de Genética, ${ }^{2}$ Departamento de Clínica Médica, \\ Faculdade de Medicina de Ribeirão Preto, Universidade de São Paulo, \\ Ribeirão Preto, SP, Brasil \\ ${ }^{3}$ Centro de Terapia Celular, CEPID/FAPESP, Centro Regional de Hemoterapia, \\ Ribeirão Preto, SP, Brasil \\ ${ }^{4}$ Applied Biosystems do Brasil, São Paulo, SP, Brasil
}

\begin{abstract}
MicroRNAs (miRNAs) are a class of small endogenous RNAs that play important regulatory roles by targeting mRNAs for cleavage or translational repression. miRNAs act in diverse biological processes including development, cell growth, apoptosis, and hematopoiesis, suggesting their association with cancer. We determined the miRNA expression profile of chronic and acute lymphocytic leukemias (CLL and ALL) using the TaqMan ${ }^{\circledR}$ MicroRNA Assays Human Panel (Applied Biosystems). Pooled leukemia samples were compared to pooled $\mathrm{CD} 19^{+}$samples from healthy individuals (calibrator) by the $2^{-\Delta \Delta \mathrm{Ct}}$ method. Total RNA input was normalized based on the $\mathrm{Ct}$ values obtained for $h s a-m i R-30 \mathrm{~b}$. The five most highly expressed miRNAs were miR-128b, miR-204, miR-218, miR-331, and miR181b-1 in ALL, and miR-331, miR-29a, miR-195, miR-34a, and miR$29 \mathrm{c}$ in CLL. To our knowledge, this is the first report associating miR128b, miR-204 and miR-331 to hematological malignancies. The miR-17-92 cluster was also found to be up-regulated in ALL, as previously reported for some types of lymphomas. The differences observed in gene expression levels were validated for miR-331 and miR-128b in ALL and CD19+ samples. These miRNAs were upregulated in ALL, in agreement with our initial results. A brief target analysis was performed for miR-331. One of its putative targets, SOCS1, promotes STAT activation, which is a known mediator of cell proliferation and survival, suggesting the possibility of an association between miR-331 and these processes. This initial screening provided information on miRNA differentially expressed in normal and malignant B-cells that could suggest the potential roles of these miRNAs in hematopoiesis and leukemogenesis.
\end{abstract}

Key words

- Non-coding RNA

- MicroRNA

- Hematopoietic malignancies

- Leukemia

- miR-331 
MicroRNAs (miRNAs) are an abundant class of short regulatory ( 22 nt) non-coding RNAs widely expressed in all metazoan eukaryotes and highly conserved throughout different organisms. miRNAs are encoded by genes that are presumably transcribed into single or clustered primary transcripts which are processed to produce the mature miRNAs. These, in turn, are incorporated into a ribonucleoprotein complex called RNA-induced silencing complex (RISC) and guide the RISC to the target mRNA. RISC plus miRNA mediate target gene down-regulation by mRNA cleavage or translational repression (1).

Human cancer is associated with changes in miRNA expression. It was recently reported that the pattern of miRNA expression varies dramatically across tumor types and that miRNA profiles reflect the developmental lineage and differentiation state of the tumor (2). miRNAs are also likely to play critical roles in various aspects of hematopoiesis including the differentiation of hematopoietic stem/progenitor cells, and in events that lead to hematological disorders (3). Nonetheless, there is still little information available about miRNA expression patterns in specific diseases.

Chronic lymphocytic leukemia (CLL) represents the most common type of leukemia and is characterized by the gradual accumulation in the patient of small mature Bcells, most of which are G0/G1 phase, nonproliferating cells and which display typical B-cell surface markers (CD19, CD20) in addition to CD5 (4).

Acute lymphocytic leukemia (ALL) is one of the most common malignancies observed in the pediatric age group. It derives from the clonal proliferation of lymphoid progenitors in the bone marrow. ALL represents about $80 \%$ of cases of acute leukemia in children and only $20 \%$ in adults. The consequence of bone marrow infiltration is the occurrence of various cytopenias in the peripheral blood associated with the appear- ance of peripheral blast cells (5).

The objective of the present study was to define a general miRNA expression profile for ALL and CLL cells compared to their normal counterparts.

The study was approved by the Ethics Committee of the Faculty of Medicine of Ribeirão Preto, University of São Paulo, and written informed consent was obtained from all patients and subjects.

Peripheral blood samples were obtained from 9 patients diagnosed with CLL on the basis of immunophenotype profile. Normal $\mathrm{CD} 19^{+}$cells were purified from peripheral blood of 6 healthy individuals. First, peripheral blood mononuclear cells were harvested by centrifugation on a Ficoll-Hypaque (GE Healthcare) density gradient. CD19+ B-cells were purified from mononuclear cells using the D19 Multisort kit (Miltenyi Biotec, Auburn, CA, USA). CLL patients were stable, at stage $\mathrm{A}$ of the Binet classification, without previous treatment and not evaluated for the mutational status of IgH VDJ genes. Normal $\mathrm{CD} 9^{+}$cells were sorted using an immunomagnetic method with anti-CD19 microbeads (BD Biosciences, San Jose, CA, USA). To evaluate cell sorting efficiency, we stained the sorted cells with an anti-CD20 fluorescein-conjugated (FITC) monoclonal antibody, and flow cytometry analysis revealed more than $95 \%$ of purity for all samples. ALL samples from the bone marrow of 7 patients diagnosed with ALL of various subtypes, including biphenotypic, pro-B, $\mathrm{T}$ and $\mathrm{B}, \mathrm{T}$ and $\mathrm{Calla}^{+}$. In order to perform the analysis, we pooled samples into three groups, ALL, CLL and normal CD19+ cells. All samples used in the present study were collected from patients who attended Hospital das Clínicas, Ribeirão Preto Medical School, University of São Paulo (HC, FMRP, USP).

We also validated the expression values of two miRNAs in ALL and CD19+ ${ }^{+}$samples individually. Total RNA from fresh samples was isolated with the Trizol LS ${ }^{\circledR}$ Reagent (Invitrogen, Carlsbad, CA, USA), according 
to the manufacturer's protocol. We used the TaqMan ${ }^{\circledR}$ MicroRNA Assays Human Panel (Applied Biosystems, PN 4365409, kindly provided by Applied Biosystems, São Paulo, SP, Brazil) to assess the expression levels of 164 miRNAs in pooled samples of B-CLL, ALL and normal CD19+ B-cells, which includes two steps: reverse transcription and real-time PCR.

Total RNA ( $2.5 \mathrm{ng} /$ reaction) from pools was reverse-transcribed with specific looped RT primers for each miRNA provided in the assay. The $10-\mu \mathrm{L}$ reactions were performed using reagents from the High-Capacity cDNA Archive Kit (PN 4322171, Applied Biosystems) and 3.8 U RNase inhibitor (PN N8080119, Applied Biosystems) and incubated for $30 \mathrm{~min}$ at $16^{\circ} \mathrm{C}, 30 \mathrm{~min}$ at $42^{\circ} \mathrm{C}, 5$ min at $85^{\circ} \mathrm{C}$, and then held at $4^{\circ} \mathrm{C}$. For the real-time PCR step, $4 \mu \mathrm{L}$ 1:4 diluted cDNA samples were used as templates in $10-\mu \mathrm{L}$ reactions containing primers and probes for each miRNA according to manufacturer instructions. All reactions were run in duplicate on an ABI7500 Sequence Detection System (Applied Biosystems, Foster City, CA, USA) using the following conditions: $95^{\circ} \mathrm{C}$ for $10 \mathrm{~min}$, followed by 40 cycles at $95^{\circ} \mathrm{C}$ for $15 \mathrm{~s}$, and $60^{\circ} \mathrm{C}$ for $1 \mathrm{~min}$. The foldchange was calculated using $2^{-\Delta \Delta \mathrm{Ct}}$ method, with nine $\mathrm{CD} 19^{+}$pooled samples from healthy individuals being used as the calibrator. Total RNA input was normalized based on the Ct values obtained for $h s a-m i R$ $30 \mathrm{~b}$, which was uniformly expressed in all three samples. Experiments with coefficients of variation greater than $5 \%$ were excluded and 75 miRNAs were left in the final analysis.

In the experimental validation step, two miRNAs were tested individually in all seven ALL and six CD19+ ${ }^{+}$samples. The miRNAs chosen for validation were miR-331 and miR-128b. We used the same real-time PCR method as used in the panel, except that total RNA input was normalized with RNU49, a nucleolar RNA currently used in this type of analysis.
We also selected the best predicted targets for a given miRNA, comparing the information from the three most known target prediction databases: TargetScan (http://www. targetscan.org/), mirBase (http://microrna. sanger.ac.uk/sequences/index. shtml), and PicTar (http://pictar.bio.nyu.edu/). This approach allowed us to select the common targets found in the three different algorithms.

The five most highly expressed miRNAs in ALL were miR-128b, miR-204, miR-218, miR-331, and miR-181b-1 (Table 1). The most represented miRNA in ALL is miR$128 \mathrm{~b}$ with a fold difference of 436.5 compared with normal CD19 ${ }^{+}$B-cells. miR-128b is a reported homologue of miR-128a and has been found in colon, lung, and pancreas solid cancers $(6,7)$. As far as we know, the involvement of these miRNAs with hematological malignancies had not been reported to date. The second most expressed miRNA in ALL was miR-204. Cheng and colleagues (8) found that inhibition of this miRNA together with eight others (miR-1d, miR-7, miR-148, miR-204, miR-210, miR-216, miR218, and miR-296) increased the level of apoptosis in HeLa cells. In our ALL samples, five of these miRNAs were hyperexpressed: miR-204 itself, miR-148, miR-210, miR218, miR-296, and miR-381 (miR-216 was hypoexpressed and miR-1d and miR-7 were not analyzed). The fourth most expressed miRNA was miR-331, which is a single miRNA located in an intergenic region of chromosome 12 (12q22) and its predicted targets are discussed below. miR-181-b is clustered with miR-213 on chromosome 1 (1q31.3) and was not found in any of the cell lines tested by $\mathrm{Yu}$ and colleagues (9). On the other hand, miR-181-a was already reported as a promoter of B-cell differentiation (9). To our knowledge, the present report is the first linking miR-128b, miR-204 and miR331 with hematological malignancies.

The miR-17-92 cluster seemed to be upregulated in all of our ALL samples. This 
Table 1. Relative expression of 46 microRNAs (miRNAs) in chronic and acute lymphocytic leukemia (CLL and ALL).

\begin{tabular}{|c|c|c|c|c|c|}
\hline miRNAs & CLL & ALL & Localization & Genetic map & Organization \\
\hline miR-015a & 1.37 & 2.12 & intergenic & $13 q 14.3$ & cluster \\
\hline miR-017-3p & 1.66 & 2.56 & intergenic & $13 q 31.3$ & cluster $^{1}$ \\
\hline miR-017-5p & 1.85 & 6.36 & intergenic & $13 q 31.3$ & cluster $^{1}$ \\
\hline $\operatorname{miR}-019 a$ & 2.56 & 6.99 & intergenic & $13 q 31.3$ & cluster $^{1}$ \\
\hline miR-020 & 3.76 & 9.06 & intergenic & $13 q 31.3$ & cluster $^{1}$ \\
\hline miR-025 & 1.34 & 3.63 & intron & $7 q 22.1$ & cluster \\
\hline miR-026b & 2.39 & 1.58 & intron & $2 q 35$ & single \\
\hline miR-027a & 2.60 & 7.11 & intergenic & $19 p 13.12$ & cluster \\
\hline miR-028 & 3.47 & 1.89 & intron & $3 q 28$ & single \\
\hline miR-029a & 27.19 & 2.10 & intergenic & $7 q 32.3$ & cluster $^{4}$ \\
\hline miR-029b & 9.92 & 0.60 & intergenic & $7 q 32.3$ & cluster 4 \\
\hline miR-029c & 8.11 & 1.59 & intergenic & $1 q 32.2$ & cluster \\
\hline miR-030a-3p & 1.08 & 4.14 & intron & $6 q 13$ & cluster \\
\hline miR-034a & 15.73 & 23.75 & intergenic & $1 p 36.23$ & single \\
\hline miR-092 & 1.86 & 5.70 & intergenic & $13 q 31.3$ & cluster $^{1}$ \\
\hline miR-096 & 2.76 & 29.96 & intergenic & $7 q 32.2$ & cluster $^{3}$ \\
\hline miR-098 & 2.06 & 3.67 & intron & Xp11.22 & cluster \\
\hline miR-099a & 0.53 & 2.06 & intron & $21 q 21.1$ & cluster \\
\hline $\mathrm{miR}-100$ & 0.89 & 4.79 & intergenic & $11 \mathrm{q} 24.1$ & cluster $^{2}$ \\
\hline miR-106a & 7.52 & 22.32 & intron & Xq26.2 & cluster \\
\hline miR-125b & 0.51 & 4.36 & intergenic & $11 \mathrm{q} 24.1$ & cluster $^{2}$ \\
\hline miR-126 & 0.70 & 3.34 & intron & $9 q 34.3$ & single \\
\hline miR-128b & 2.06 & 436.55 & intron & 3p22.3 & single \\
\hline miR-130a & 0.65 & 6.94 & intergenic & $11 q 12.1$ & single \\
\hline miR-130b & 1.78 & 21.33 & intergenic & $22 q 11.21$ & single \\
\hline $\mathrm{miR}-146$ & 1.89 & 8.51 & intergenic & $5 q 33.3$ & single \\
\hline miR-148a & 7.01 & 4.42 & intergenic & 7p15.2 & single \\
\hline $\mathrm{miR}-150$ & 2.58 & 0.14 & 3'UTR & $19 q 13.33$ & single \\
\hline miR-152 & 2.01 & 1.04 & intron & $17 q 21.32$ & single \\
\hline miR-181b & 1.22 & 37.53 & intergenic & $1 q 31.3$ & cluster \\
\hline miR-181c & 0.14 & 18.96 & intergenic & $19 p 13.12$ & cluster \\
\hline miR-182 & 2.09 & 7.01 & intergenic & $7 q 32.2$ & cluster $^{3}$ \\
\hline miR-185 & 0.10 & 2.06 & intron & $22 q 11.21$ & single \\
\hline miR-191 & 0.73 & 5.58 & intron & $3 p 21.31$ & cluster \\
\hline miR-194 & 1.50 & 2.33 & intron & $1 q 41$ & cluster \\
\hline miR-195 & 20.39 & 33.59 & intergenic & $17 \mathrm{p} 13.1$ & cluster \\
\hline$m i R-199 b$ & 0.77 & 3.29 & intron & $9 q 34.11$ & single \\
\hline miR-210 & 1.72 & 2.79 & intergenic & $11 p 15.5$ & single \\
\hline miR-296 & 2.02 & 1.00 & intergenic & $20 q 13.32$ & single \\
\hline miR-301 & 1.40 & 6.94 & intron & $17 q 22$ & single \\
\hline miR-320 & 0.90 & 6.52 & 5'UTR & $8 p 21.3$ & single \\
\hline miR-324-5p & 1.30 & 3.40 & intron & $17 p 13.1$ & single \\
\hline miR-331 & 40.93 & 74.03 & intergenic & $12 q 22$ & single \\
\hline miR-335 & 1.82 & 2.32 & intron & $7 q 32.2$ & single \\
\hline miR-340 & 0.84 & 2.54 & intron & $5 q 35.3$ & single \\
\hline Let7b & 0.26 & 2.25 & intergenic & $22 q 13.31$ & cluster \\
\hline
\end{tabular}

The numbers reported are fold values relative to the value obtained for the pool of CD19+ control samples from normal donors.

$1,2,3$ Members of the same cluster; ${ }^{4}$ miRNAs that are not clustered. cluster consists of miR-17-3p, miR-17-5p, miR-18, miR-19a, miR-20, miR-19b-1, and miR-92. Of these, only miR-18 and miR19b-1 were not analyzed and all the others were up-regulated in our ALL samples. We also detected miR-106a, a member of a homologous cluster on chromosome X (miR106a-92). This cluster is located on chromosome 13 (13q31-32), a genomic locus that is amplified in some types of lymphomas. Under experimental conditions, this amplification resulted in overexpression of the miRNA species from the miR-17-92 cluster, strongly favoring transformation of B-cell progenitors (10). Since this miRNA cluster is directly regulated by c-Myc, the expression of these miRNAs may promote tumorigenesis, and this activity might be responsible, in part, for apoptosis suppression (11).

In ALL, the four miRNAs with lowest expression levels when compared with control CD19+ ${ }^{+}$-cells were miR-135b, miR132, miR-199s, miR-139, and miR-150. miR$135 \mathrm{~b}$ has been reported to be up-regulated in colorectal cancer cell lines and tumor samples (12). miR-132 is a brain-enriched miRNA located in an intergenic region of chromosome 17, clustered with miR-212 (13). miR199s, also known as miR-199-a-1, was recently found in a human fetal liver miRNA library (14). miR-199s was also down-regulated in colorectal cancer cell lines (12).

In CLL samples, the five most highly expressed miRNAs were miR-331, miR-29a, miR-195, miR-34a, and miR-29c (Table 1). miR-331 was the highest represented miRNA in CLL (40.9-fold) and the fourth in ALL, as already discussed above. The second most represented miRNA was miR-29a (27.2fold), which is clustered with miR-29b-1. Recently, this cluster was identified as a hematopoietic-enriched cluster in Jurkat (human acute T-cell leukemia) and CMK (human megakaryoblastic leukemia) cell lines (9). miR-195 was the third most represented miRNA (20.4-fold) in CLL and seems to be clustered with miR-497 on chromosome 17. 
miR-34a and miR-29c were up-regulated in our CLL samples (15.7- and 8.1-fold, respectively) and their precursors were previously reported to be up-regulated in CLL samples (15).

The five miRNAs with lowest expression levels in CLL were miR-135b, miR199s, miR-142-5p, miR-185, and miR-181c. miR-142-5p was identified as a hematopoietic-specific miRNA together with miR-223 and miR-181 (16). Expression of miR-142 was present in normal human T-cells and granulocytes but weak in monocytes and Bcells. In human hematopoietic cell lines (ALL, T-ALL, various types of lymphomas, acute myelogenous lymphoma, chronic myelogenous lymphoma, and others), this miRNA was only weakly detected (17). The $\mathrm{t}(8 ; 17)$ translocation was identified in aggressive B-cell disorders, causing the fusion of the promoter and 5'portion of the miR142 gene to the truncated $m y c$ gene. The fusion gene contains the intact miR-142 stemloop precursor, but the miR-142-3' flanking sequence is replaced with the truncated myc gene. It has not been determined whether this gene fusion leads to overproduction of the truncated MYC protein, to the disruption of miR-142 maturation, or to both, but it would be of great interest to determine the expression levels of this miRNA in patients with confirmed $\mathrm{t}(8 ; 17)$ translocation.

In agreement with Fulci and colleagues (18), we found an inverse correlation between miR-142-5p, which is down-regulated in CLL $\left(2^{-\Delta \Delta \mathrm{Ct}}=0.092\right)$ and $\mathrm{miR}-142-3 \mathrm{p}$, which is up-regulated in CLL $\left(2^{-\Delta \Delta \mathrm{Ct}}=1.44\right)$. miR-185 was already reported to be downregulated in some solid tumors (7). In our study, miR-181c was hypoexpressed in CLL, although its expression in normal CD19+ $\mathrm{B}$ cells was also low $\left(2^{\wedge}-\mathrm{ddCt}=0.139\right)$. miR$181 \mathrm{c}$ has been found to be hyperexpressed in two T-cell lines, Jurkat and Hut-78, and has not been detected in a B-cell line (3D5) (9).

We have chosen two miRNAs to validate our expression measurements, miR-331 and
miR-128b for ALL and CD19+ samples only. All ALL samples overexpressed these two miRNAs when compared to $\mathrm{CD} 19^{+}$cells, corroborating the results obtained for the pooled samples in the initial screening. The results are shown on Figure 1. T-ALL and pro-B ALL samples showed the highest expression values for miR-128b, while the two biphenotypic ALL samples showed the lowest expression values. miR-331 showed little variation among samples.

We also performed a predicted target analysis for miR-331, which was chosen because it is overexpressed in both types of leukemia and because there is still little information about its expression and function. Results from three target databases were compared for this target analysis: mirBase (miRanda), TargetScan and PicTar. Only those targets which were common to the three target databases were evaluated. Among predicted targets, we found one which can be directly related to cytokine signaling, SOCS1 (suppressor of cytokine signaling). Loss of function of this protein is involved in the development and progression of several specific cancers, because SOCS1 exerts the function of a negative feedback regulator of STAT action, and the latter is involved in cellular processes including proliferation, cell survival and angiogenesis (19). Constitutive activation of the STAT indicating altered JAK/STAT signaling has been reported for several lymphomas including classical Hodgkin's lymphoma and primary mediastinal Bcell lymphoma (20). Therefore, it is possible

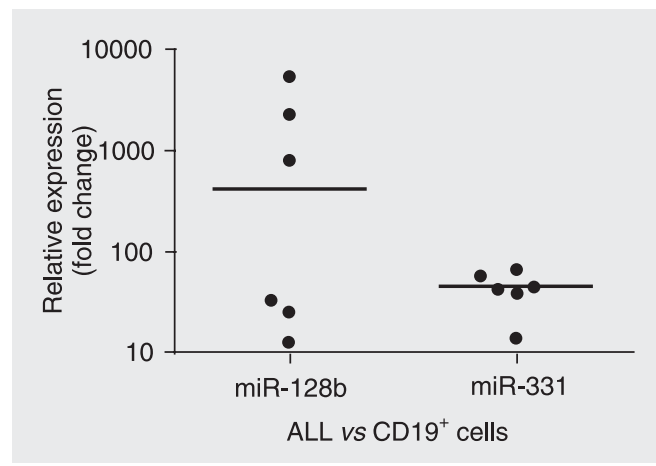

Figure 1. Expression values for miR-331 and miR-128b in six acute lymphocytic leukemia (ALL) samples analyzed individually, to validate the results found for those pooled samples. The fold change is the $2^{-\Delta \Delta C t}$ value, calculated with the mean $\Delta \mathrm{Ct}$ value obtained for CD19+ samples (calibrator). 
that miRNAs might have a role in the loss of function of SOCS1.

This initial screening provided general data on miRNA expression in ALL and CLL. The expression levels of the miRNAs already reported to be expressed in lymphoid cells were confirmed and the differences between normal and malignant B-cells could point to the potential roles for these miRNAs in normal hematopoiesis and leukemogenesis. Changes of miRNA expression levels may play an important role in the genesis and evolution of lymphoid malignancies. Analysis of putative targets of miR-331 supports the idea that its overexpression may be related to increased cell proliferation and survival by means of SOCS1 down-regulation. Equally important, changes of apopto- sis levels are a central mechanism for disease. Another miRNA that may be involved in the pathogenesis of ALL is the mir-17-92 cluster. Although the exact cellular role for this cluster is unknown, its up-regulation may reduce apoptosis in ALL, as demonstrated for some types of lymphomas, where its overexpression could change the cell potential for survival.

\section{Acknowledgments}

The authors would like to thank Amélia Góes Araújo, Marli H. Tavela, Lidiane Cristina Melato, and Anemari Ramos Dinarte dos Santos for assistance with the laboratory techniques.

\section{References}

1. Novina CD, Sharp PA. The RNAi revolution. Nature 2004; 430: 161164.

2. Lu J, Getz G, Miska EA, Alvarez-Saavedra E, Lamb J, Peck D, et al. MicroRNA expression profiles classify human cancers. Nature 2005; 435: 834-838.

3. Chen CZ, Lodish HF. MicroRNAs as regulators of mammalian hematopoiesis. Semin Immunol 2005; 17: 155-165.

4. Cheson BD, Bennett JM, Grever M, Kay N, Keating MJ, O'Brien S, et al. National Cancer Institute-sponsored Working Group guidelines for chronic lymphocytic leukemia: revised guidelines for diagnosis and treatment. Blood 1996; 87: 4990-4997.

5. Wiernick P, Canellos G, Dutcher J, Kyle R. Neoplastic diseases of the blood. 3rd edn. New York: Churchill Livingstone Incorporation; 1996.

6. Lim LP, Glasner ME, Yekta S, Burge CB, Bartel DP. Vertebrate microRNA genes. Science 2003; 299: 1540.

7. Volinia S, Calin GA, Liu CG, Ambs S, Cimmino A, Petrocca F, et al. A microRNA expression signature of human solid tumors defines cancer gene targets. Proc Natl Acad Sci U S A 2006; 103: 22572261.

8. Cheng AM, Byrom MW, Shelton J, Ford LP. Antisense inhibition of human miRNAs and indications for an involvement of miRNA in cell growth and apoptosis. Nucleic Acids Res 2005; 33: 1290-1297.

9. Yu J, Wang F, Yang GH, Wang FL, Ma YN, Du ZW, et al. Human microRNA clusters: genomic organization and expression profile in leukemia cell lines. Biochem Biophys Res Commun 2006; 349: 59-68.

10. He L, Thomson JM, Hemann MT, Hernando-Monge E, Mu D, Goodson $\mathrm{S}$, et al. A microRNA polycistron as a potential human oncogene. Nature 2005; 435: 828-833.

11. Mendell JT. MicroRNAs: critical regulators of development, cellular physiology and malignancy. Cell Cycle 2005; 4: 1179-1184.

12. Bandres E, Cubedo E, Agirre X, Malumbres R, Zarate R, Ramirez N, et al. Identification by real-time PCR of 13 mature microRNAs differentially expressed in colorectal cancer and non-tumoral tissues. $\mathrm{Mol}$ Cancer 2006; 5: 29.

13. Sempere LF, Freemantle S, Pitha-Rowe I, Moss E, Dmitrovsky E, Ambros V. Expression profiling of mammalian microRNAs uncovers a subset of brain-expressed microRNAs with possible roles in murine and human neuronal differentiation. Genome Biol 2004; 5: R13.

14. Fu H, Tie Y, Xu C, Zhang Z, Zhu J, Shi Y, et al. Identification of human fetal liver miRNAs by a novel method. FEBS Lett 2005; 579: 38493854.

15. Calin GA, Liu CG, Sevignani C, Ferracin M, Felli N, Dumitru CD, et al. MicroRNA profiling reveals distinct signatures in $\mathrm{B}$ cell chronic lymphocytic leukemias. Proc Natl Acad Sci U S A 2004; 101: 1175511760.

16. Chen C-Z, Ling L, Lodish HF, Bartel DP. MicroRNAs modulate hematopoietic lineage differentiation. Science 2004; 303: 83-86.

17. Ramkissoon SH, Mainwaring LA, Ogasawara $\mathrm{Y}$, Keyvanfar $\mathrm{K}$, McCoy JP Jr, Sloand EM, et al. Hematopoietic-specific microRNA expression in human cells. Leuk Res 2006; 30: 643-647.

18. Fulci V, Chiaretti S, Goldoni M, Azzalin G, Carucci N, Tavolaro S, et al. Quantitative technologies establish a novel microRNA profile of chronic lymphocytic leukemia. Blood 2007; 109: 4944-4951.

19. Rakesh K, Agrawal DK. Controlling cytokine signaling by constitutive inhibitors. Biochem Pharmacol 2005; 70: 649-657.

20. Weniger MA, Melzner I, Menz CK, Wegener S, Bucur AJ, Dorsch K, et al. Mutations of the tumor suppressor gene SOCS-1 in classical Hodgkin lymphoma are frequent and associated with nuclear phospho-STAT5 accumulation. Oncogene 2006; 25: 2679-2684. 